\title{
When Should Statins Be Stopped?
}

\author{
Mário Bibi, Ana Ferro, Filipa Guimarães, Paulo Coelho, Inês Chora \\ Hospital Pedro Hispano, Sra. da Hora, Portugal
}

Received: 20/05/2021

Accepted: 25/05/2021

Published: $15 / 06 / 2021$

How to cite this article: Bibi M, Ferro A, Guimarães F, Coelho P, Chora I. When should statins be stopped? EJCRIM 2021;8: doi:10.12890/2021_002661.

Conflicts of Interests: The authors declare there are no competing interests.

This article is licensed under a Commons Attribution Non-Commercial 4.0 License

\section{ABSTRACT}

Mycobacterium chelonae is a non-tuberculous mycobacterium that can cause skin infections in immunocompetent individuals. We report a case of skin infection by this agent in a woman with dyslipidaemia, that culminated in statin-induced rhabdomyolysis due to the combination of clarithromycin, ciprofloxacin and simvastatin.

\section{LEARNING POINTS}

- Skin infection with Mycobacterium chelonae is an increasing global problem among immunocompetent individuals.

- Statin-induced rhabdomyolysis is an important and avoidable end-result of drug-drug interaction.

- Inhibition of cytochrome P450 isoenzyme 3A4 and of organic anion transporting polypeptide $1 B 1$ are two important examples of statin interference with metabolism, and clarithromycin can inhibit both.

\section{KEYWORDS}

Mycobacterium chelonae, statin-induced rhabdomyolysis, ciprofloxacin, clarithromycin

\section{CASE DESCRIPTION}

A 67-year-old woman with type 2 diabetes mellitus, essential arterial hypertension, dyslipidaemia and atrial fibrillation was being followed for basal cell carcinoma. She regularly used the water of a private well for drinking and washing. She reported no alcohol intake. She was medicated with simvastatin $40 \mathrm{mg} /$ day for 2 years prior to the events described below, enalapril, furosemide, gliclazide, metformin and warfarin. In a follow-up consult, a nodular, erythematous and infiltrative lesion on the dorsal aspect of the left hand was found (Fig. 1). Biopsy results were compatible with cutaneous mycobacterial infection (granulomatous inflammatory process, with central necrosis and the presence of acid-alcohol resistant bacillus), and Mycobacterium chelonae was identified. Whole-body computed tomography was unremarkable. Antibiotic therapy was initiated, with ciprofloxacin 500 mg twice daily and clarithromycin 500 mg twice daily. One week later, the patient developed generalized muscular weakness, mainly proximal, myalgia, nausea and aqueous diarrhoea and attended our Emergency Department. At admission, she was dehydrated, with global diminished muscular strength (grade 4/5 on the Medical Research Council manual muscle testing scale), tenderness at muscular palpation and hard oedema of the extremities. She had an otherwise normal physical and neurological exam.

The initial work-up showed elevated serum creatine kinase (17,830 U/I) as well as aspartate transaminase (1,003 $\mathrm{U} / \mathrm{I})$ and alanine transaminase $(556 \mathrm{U} / \mathrm{l})$, that were interpreted in the context of rhabdomyolysis. The serum creatinine level was $2.3 \mathrm{mg} / \mathrm{dl}$, associated with metabolic acidosis and mild hyperkalaemia; the remaining electrolytes were normal. Abdominal ultrasound showed no liver or urinary system changes. The patient was hospitalized and fluid administration was started. Statin and antibiotics were stopped.

Muscle biopsy showed rare atrophic fibres without necrotic or regenerative fibres, and no inflammatory infiltrates or vasculitic lesions. Electromyography (EMG) findings were compatible with a myopathy. An autoimmune testing panel was negative. Serologies for syphilis, 
hepatitis B and C, human immunodeficiency virus and Borrelia were negative. Serologies for Epstein-Barr, cytomegalovirus, herpes simplex 1 and 2 were negative for acute infection. Thyroid hormones were within normal levels.

The patient exhibited a slow but total recovery with only supportive measures. After 3 weeks, rhabdomyolysis was substantially better (Fig. 2), with normalization after 6 months, associated with normal muscular strength and EMG.

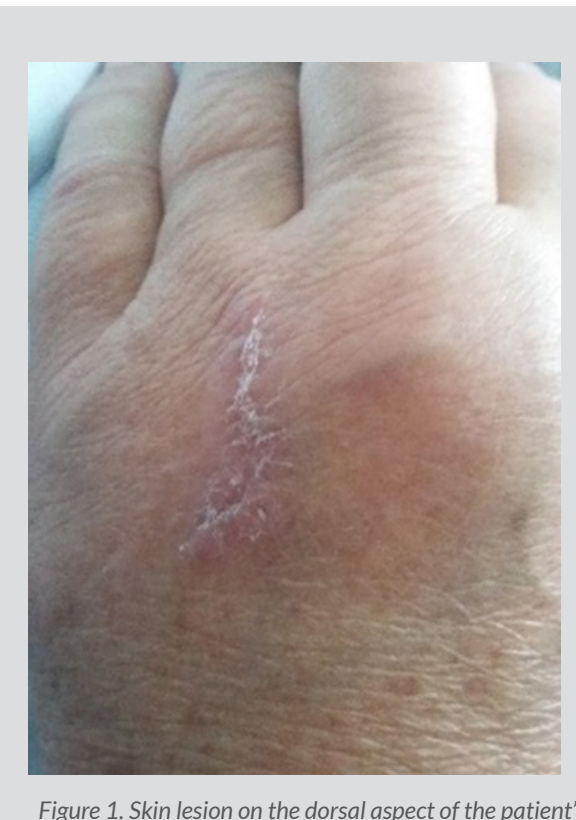

left hand

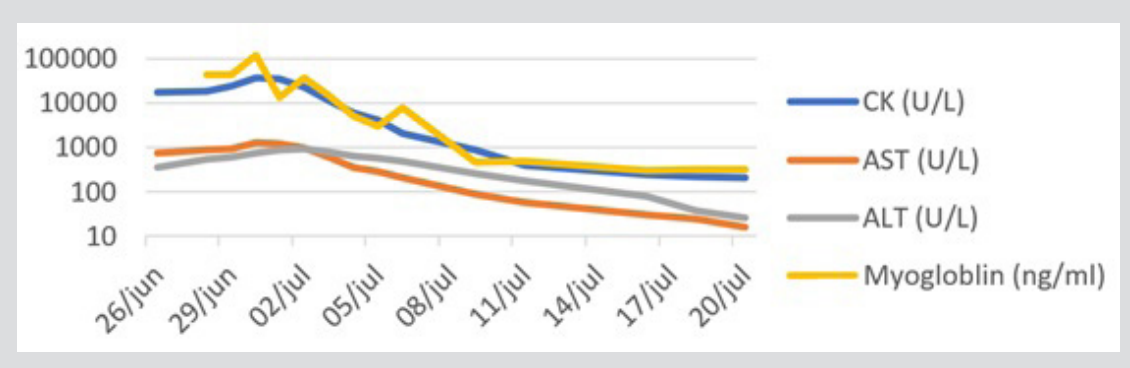

Figure 2. Rhabdomyolysis evolution.

$A L T$, alanine transaminase; $A S T$, aspartate transaminase; $C K$, creatinine kinase

One month after admission, the patient restarted antibiotic therapy with doxycycline $200 \mathrm{mg}$ daily and moxifloxacin $400 \mathrm{mg}$ daily. The antibiotic combination was prolonged for 9 months, with clinical resolution of the hand lesion and no new lesions formed. Statin was only reintroduced after antibiotic termination. During the 2 years of follow-up, the patient exhibited no new muscular events.

\section{DISCUSSION}

M. chelonae is a non-tuberculous mycobacterium which can be found in the soil, water and aquatic animals. The global incidence of infection with this mycobacterium is reportedly increasing ${ }^{[1]}$. In immunocompetent individuals, $M$. chelonae can cause localized skin infections, as in our patient. There are no descriptions in the literature of generalized myopathy in immunocompetent patients caused by this agent. For correct diagnosis, a skin biopsy is needed for histopathological examination, including acid-fast staining and mycobacterial culture. Guided by susceptibility testing, combination therapy with at least two antibiotic agents, for a minimum of 4 months for skin disease, is recommended ${ }^{[1]}$.

Rhabdomyolysis is a condition resulting from muscle injury and involves necrosis of muscle tissue that leads to the release of intracellular content into the blood stream. Typical clinical findings include muscle weakness, pain and dark tea-coloured urine. CK elevation more than 10 times the upper limit of normal or above 1,000 U/I is diagnostic. The management of rhabdomyolysis relies on treating/removing the underlying cause of muscle injury and preventing acute kidney injury (AKI). The cornerstone intervention to prevent AKI is fluid administration ${ }^{[2]}$.

The case presented is typical of a drug-induced rhabdomyolysis, given the temporal correlation, subsequent evolution with only supportive measures, and the absence of trauma, infection or autoimmune disease. Statins have been associated with rhabdomyolysis ${ }^{[3]}$. Almost $50 \%$ of cases of statin-induced rhabdomyolysis are precipitated by another drug that interferes with statin metabolism, increasing its concentration. Inhibition of the cytochrome P450 isoenzyme 3A4 (CYP3A4) plays a major role in most cases of statin-induced rhabdomyolysis. Statins metabolized by CYP3A4 include atorvastatin, simvastatin and lovastatin ${ }^{[3]}$. Both clarithromycin and ciprofloxacin are known CYP3A4 inhibitors and have separately been implicated in statin-induced rhabdomyolysis in case reports ${ }^{[4,5]}$. Clarithromycin is also an inhibitor of organic anion transporting polypeptide 1B1 (OATP1B1), a transporter protein involved in the metabolism pathway of all statins, including those not metabolized by CYP3A4 ${ }^{[3]}$. 
When a patient is on simvastatin, atorvastatin or lovastatin, drugs with potential CYP3A4 inhibition should be avoided and alternatives considered. If no equivalent alternatives to a CYP3A4 inhibitor exist (Table 1), temporary suspension of simvastatin, atorvastatin or lovastatin or a switch to a non-CYP3A4-metabolized statin should be considered. Attention should also be directed to OATP1B1 inhibitors given the potential interaction with all statins ${ }^{[3]}$. The main complication of maintaining statins in a potential drug-drug interaction is statininduced rhabdomyolysis, with potentially devastating renal consequences, as seen in our case. Ultimately, it can lead to death if the statin is not stopped in time. In a world of increasing polypharmacy, identifying the possibility of drug-drug interaction is of major importance in order to prevent unwanted effects.

\begin{tabular}{|l|l|l|}
\hline Amiodarone & Amlodipine & Cimetidine \\
\hline Ciprofloxacin & Clarithromycin* & Cyclosporine* \\
\hline Diltiazem & Fluconazole & Fluoxitine \\
\hline Grapefruit & Imatinib & Isoniazid \\
\hline Itraconazole & Midalozam & Posaconazole \\
\hline Protease inhibitors* & Ranolazine & Sertraline \\
\hline Tacrolimus & Ticagrelor & Tricyclic antidepressants \\
\hline
\end{tabular}

Table 1. Known CYP3A4 inhibitors (adapted from Wiggins et al [1].

*Simultaneous inhibition of CYP3A4 and OATP1B1.

\section{REFERENCES}

1. Uslu U, Böhm O, HepptF, Sticherling M. Skin and soft tissue infections caused by Mycobacterium chelonae: more common than expected? Acta Derm Venereol 2019;99(10):889893.

2. Cabral BMI, Edding SN, Portocarrero JP, Lerma EV. Rhabdomyolysis. Dis Mon 2020;66(8):101015.

3. Wiggins BS, Saseen JJ, Page RL 2nd, Reed BN, Sneed K, Kostis JB, et al; American Heart Association Clinical Pharmacology Committee of the Council on Clinical Cardiology; Council on Hypertension; Council on Quality of Care and Outcomes Research; and Council on Functional Genomics and Translational Biology. Recommendations for management of clinically significant drug-drug interactions with statins and select agents used in patients with cardiovascular disease: a scientific statement from the American Heart Association. Circulation 2016;134(21):e468-e495.

4. Patel AM, Shariff S, Bailey DG, Juurlink DN, Gandhi S, Mamdani M, et al. Statin toxicity from macrolide antibiotic coprescription: a population-based cohort study. Ann Intern Med 2013;158(12):869-876.

5. Goldie FC, Brogan A, Boyle JG. Ciprofloxacin and statin interaction: a cautionary tale of rhabdomyolysis. BMJ Case Rep 2016;2016:bcr2016216048. 
European Journal

of Case Reports in

Internal Medicine 